\title{
The Voronovskaja-type formula for the Bleimann, Butzer and Hahn operators
}

\author{
DAN BĂRBOSU, ANA MARIA ACU and FLORIN D. SOFONEA
}

\section{ABSTRACT.}

We present a short and simple proof to the Voronovskaja-type formula for the Bleimann, Butzer and Hahn operators. The proof is essentially based on the relationship between the Bleimann, Butzer and Hahn operators and the Stancu operators.

\section{REFERENCES}

[1] Abel, U., On the asymptotic approximation with operators of Bleimann,Butzer and Hahn, Indag. Math. (N.S.), 7 (1996), 1-9

[2] Abel, U. and Ivan, M., Some identities for the operator of Bleimann, Butzer and Hahn involving divided differences, Calcolo, 36 (1999), 143-160

[3] Agratini, O., Approximare prin operatori liniari, Presa Univ. Clujeană, Cluj-Napoca, 2000

[4] Bărbosu, D., Simultaneous approximation by Schurer-Stancu type operators, Math. Balkanica, 17 (2003), Fasc. 3-4, 1-6

[5] Bărbosu, D., Polynomial approximation by means of Schurer-Stancu type operators, Ed. Univ. de Nord, Baia Mare, 2006

[6] Bernstein, S. N., Demonstration du théoreme de Weierstrass fondée sur le calcul des probabilités, Commun. Soc. Math. Kharkow, 13 (1912-1913), No. $2,1-2$

[7] Bleimann, B., Butzer, P. L. and Hahn, L., A Bernstein-type operator approximating continuous functions on the semi-axis, Nederl. Akad. Wetensch. Indag. Math., 42 (1980), 255-262

[8] Della Vecchia, B., Some properties of a rational operator of Bernstein-type, In : Nevai, P., Pinkus, A.,(eds) : Progress in approximation theory, Boston, Academic Press 1991, 177-185

[9] Ivan, M., A note on the Bleimann, Butzer and Hahn operator, Automat, Comput. Appl. Math., 47 (1997), 267-272

[10] Ivan, M., Elements of Interpolation Theory, Mediamira Science Publisher, Cluj-Napoca (2004)

[11] Mercer, A. McD., A Bernstein-type operator of Bleimann, Butzer and Hahn, J. Approx. Theory, 53 (1988), 295-303

[12] Stancu, D. D., Asupra unei generalizări a polinoamelor lui Bernstein, Studia Univ. "Babeş-Bolyai", 14 (1969), No. 2, 31-45

[13] Totik, V., Uniform approximation by Bernstein-type operators, Nederl. Akad. Wetensch. Indag. Math., 46 (1984), No. 1, 87-93

[14] Voronovskaja, E., Détermination de la forme asymptotique d'approximation des fonctions par les polynômes de S. N. Bernstein, C. R. Acad. Sci. URSS (1932), 79-85

DePARTMENT OF MATHEMATICS AND COMPUTER SCIENCE

FACUlty OF SCIENCES NORTH University CENTER AT Baia MaRe

TECHNICAL UNIVERSITY OF ClUJ-NAPOCA

VictORIEI 76, 430122 BAIA MARE, ROMANIA

E-mail address: barbosudan@yahoo.com

UNIVERSity "Lucian Blaga" OF Sibiu

DEPARTMENT OF MATHEMATICS AND INFORMATICS

DR. I. RATIU 5-7, 550012 - SIBIU, ROMANIA

E-mail address: acuana 77@yahoo.com

E-mail address: florin.sofonea@ulbsibiu.ro

Received: 29.05.2014; In revised form: 14.10.2014; Accepted: 15.10.2014

2010 Mathematics Subject Classification. 41A25, 41A36 .

Key words and phrases. Bernstein operators, Bleimann - Butzer - Hahn (BBH) operators, Stancu operators, Voronovskaja-type formula. 